\title{
DYNAMIC GAME OF OFFENDING AND LAW ENFORCEMENT: A STOCHASTIC EXTENSION
}

\author{
DAVID W.K. YEUNG \\ SRS Consortium for Advanced Study in Cooperative Dynamic Games, Hong Kong Shue Yan University \\ Center of Game Theory, St Petersburg State University, St Petersburg, 198904, Russia \\ dwkyeung@hksyu.edu \\ YINGXUAN ZHANG \\ Department of Urban Planning \& Design, University of Hong Kong \\ cyxzhang@hku.hk \\ PATRICIA M. YEUNG \\ Faculty of Dentistry, University of Hong Kong \\ yeungpm@hku.hk \\ DEMI MAK \\ Department of Criminal Justice, New Mexico State University \\ demigolf@hotmail.com
}

\begin{abstract}
This note provides an extension of the Fend-Feichtinger-Tragler dynamic game of offending and law enforcement to a stochastic framework. This allows the analysis to reflect actual crime statistics which displays randomness in its distribution. Stochastic paths of crimes are derived. The asymptotic stationary distribution of crime records is also obtained.
\end{abstract}

Keywords: Economics of Crime; Law Enforcement; Stochastic Differential Game.

\section{Introduction}

Fent et al (2002) analyze a differential game describing the interactions between a potential offender and the law enforcement agency. However, actual crime statistics displays randomness in its distribution as shown in the Appendix. To generate this effect we extend the Fend-Feichtinger-Tragler game in Fent et al (2002) to a stochastic differential game. Stochastic dynamics are derived. This note is organized as follows. In Section 2, an intertemporal game played between an offender and a law enforcer with stochastic dynamics is set up. Analysis on the outcome of the game is performed and the solution crime evolution path is derived. Section 4 considers the case when the time horizon approaches infinity. The stationary distribution of crime records is obtained. Concluding remarks are given in Section 5.

\section{A Stochastic Fend-Feichtinger-Tragler Game of Law Enforcement}


Following Fent et al (2002), we consider the behaviour of two rational players. The first player is a (group of) potential offender who tries to maximize profit gained from illegal activities, while the second player is a law enforcement agency that tries to maximize public welfare. The offender's decision variable is $u_{1}(t)$, the rate of offence, and the agency chooses its rate of crime investigation $u_{2}(t)$.

As argued in Fent et al (2002), it may be less plausible why the authority should concentrate its activities -- and therefore its spending on investigation, prosecution, and execution of sentences -- on one particular offender. The state $x(t)$ represents the offender's experience in committing crimes or her record of prior criminal offences. The state variable has two possible interpretations. First, it can be considered as the record of prior crimes. Following Greenwood et al (1994), it is assumed that the increase of this record only depends on the criminal activity and the number of convictions, but not on the punishment. A decay term $-\delta x$ describes those cases in which former crimes are only considered for a limited period. The second interpretation is to regard $x$ as the offender's level of experience. The experience increases in proportion to the intensity $u_{1}$. However, the offender also forgets, and the experience decays with time due to changes of law and technology. Thus, the value of experience will be reduced by a rate $\delta$.

To introduce randomness we use the stochastic differential equation

$$
d x(t)=\left(u_{1}-\delta x\right) d t+\varepsilon x d z(t)
$$

to describes the dynamics of the state $x$. In particular, where $\varepsilon$ is a constant and $z(s)$ is a Wiener process and the initial state $x_{0}$. Equation (2.1) shows that the decay rate $\delta$ is subject to stochastic shocks.

The utility the offender obtains from criminal activities consists of revenues minus costs. The revenues of the offender is

$$
R\left(u_{1}\right)=\gamma u_{1} \text {. }
$$

The offender's costs consist of two terms, $S$ and $C$. The term $S$ represents the costs connected with the sentence. These costs depend on the decision variables $u_{1}$ and $u_{2}$ and the state $x$ :

$$
S\left(x, u_{1}, u_{2}\right)=\pi\left(u_{1}, u_{2}\right)+\sigma(x)=u_{1} u_{2}+\varphi x .
$$


In particular, the punishment an offender will suffer is a function of his own offending intensity, the rate of crime investigation, and the criminal record. The offence level $u_{1}$ influences the probability of being convicted and the level of punishment, the investigation activities $u_{2}$ affect the probability of being convicted and prosecuted, and the criminal record $x$ influences the level of punishment. With the formulation in (2.3), the function $S\left(x, u_{1}, u_{2}\right)$ takes positive values in case of a criminal record $x>0$ even if $u_{1}=0$. This represents, for example, the disadvantages one might experience in the labour market after having been convicted.

The second cost term $C$ represents costs that are not related to incarceration or conviction. These costs are increasing in the criminal intensity the following costfunction is adopted.

$$
C\left(u_{1}\right)=\psi u_{1}^{2} .
$$

At terminal time $T$, there is a salvage value function $Q_{1}(x(T))$ which assesses the value of the state $x$ at the end of the planning period. It represents the damage or harm (or utility) caused to the offender by having the criminal record $x(T)$. It is assumed that

$$
Q_{1}(x(T))=-\eta x(T) .
$$

If the criminal record $x(T)$ has a negative impact, $\eta$ must be positive. In the case where the offender does not consider a criminal record to be something bad at all, the parameter $\eta$ can be equal to or less than zero.

The offender's expected objective functional can be expressed as:

$$
J_{1}=E\left\{\int_{0}^{T} e^{-r t}\left[\mathcal{u}_{1}-u_{1} u_{2}-\varphi x-\psi u_{1}^{2}\right] d t-e^{-r_{1} T} \eta x(T)\right\},
$$

where $r$ is the discount rate.

The expected objective functional of the law enforcement agency is

$$
J_{2}=E\left\{-\int_{0}^{T} e^{-r t}\left[D\left(u_{1}\right)+K\left(x, u_{2}\right)+v x+L\left(u_{1}, u_{2}\right)\right] d t\right\}
$$


where all terms are costs for the state. Since the criminal record and the experience of a previously convicted offender at the terminal time do not influence public welfare there is no salvage value of the state $x$.

The damage $D\left(u_{1}\right)$ caused by illegal activities increases with the offence rate and it is assumed:

$$
D\left(u_{1}\right)=\zeta u_{1}^{2} .
$$

The costs of law enforcement, $K\left(x, u_{2}\right)$, increase and have non-decreasing marginal costs. More experienced offender might be more difficult to arrest but, on the other hand, the higher the level of criminal experience already is, the smaller the advantage of one additional unit of experience. The law enforcement cost is assumed to be:

$$
K\left(x, u_{2}\right)=\vartheta u_{2}^{2}+v x .
$$

The term $L\left(u_{1}, u_{2}\right)$ in the law enforcement agency's objective functional reflects the costs of imposing a certain punishment. For instance, the costs implied by maintaining prisons might be included here. In particular:

$$
L\left(u_{1}, u_{2}\right)=\omega u_{1} u_{2} .
$$

The expected objective functional of the law enforcement agency in (2.6) can then be expressed as:

$$
J_{2}=E\left\{-\int_{0}^{T} e^{-r t}\left[\zeta u_{1}^{2}+\vartheta u_{2}^{2}+v x+\omega u_{1} u_{2}\right] d t\right\} .
$$

\section{Analysis}

The game (2.1), (2.5) and (2.7) is a stochastic version of the Fend-Feichtinger-Tragler game. Given the presence of stochasticity, a feedback solution has to be sought. A Nash equilibrium solution for this stochastic differential game can be characterized as:

Theorem 3.1. A pair of feedback strategies $\left\{\phi_{1}^{*}(t, x) ; \phi_{1}^{*}(t, x)\right\}$ provides a Nash equilibrium solution to the game (2.1), (2.5) and (2.7) if there exist suitably smooth 
functions $V^{i}(t, x):[0, T] \times R \rightarrow R, i \in\{1,2\}$, satisfying the partial differential equations

$$
\begin{aligned}
& -V_{t}^{1}(t, x)-\frac{1}{2} \sigma^{2} x^{2} V_{x x}^{1}(t, x)=\max _{u_{1}}\left\{e^{-r t}\left[\mu_{1}-u_{1} \phi_{2}(t, x)-\varphi x-\psi u_{1}^{2}\right]\right. \\
& \left.+V_{x}^{1}(t, x)\left(u_{1}-\delta x\right)\right\}, \\
& V^{1}(T, x)=-e^{-r T} \eta x ; \\
& -V_{t}^{2}(t, x)-\frac{1}{2} \sigma^{2} x^{2} V_{x x}^{2}(t, x)=\max _{u_{2}}\left\{-e^{-r t}\left[\zeta\left(\phi_{1}(t, x)\right)^{2}+\vartheta u_{2}^{2}+v x+\omega \phi_{1}(t, x) u_{2}\right]\right. \\
& \left.+V_{x}^{2}(t, x)\left[\phi_{1}(t, x)-\delta x\right]\right\}
\end{aligned}
$$

$V^{2}(T, x)=0$.

Proof. Follow the proof of Theorem 2.5.1 in Yeung and Petrosyan (2006). $\square$ Performing the indicated maximization in (3.1) yields the conditions:

$$
\begin{aligned}
& \gamma-\phi_{2}(t, x)-2 \psi \phi_{1}(t, x)+e^{r t} V_{x}^{1} \leq 0, \\
& u_{1}\left[\gamma-\phi_{2}(t, x)-2 \psi \phi_{1}(t, x)+e^{r t} V_{x}^{1}\right]=0 ; \\
& -2 \vartheta \phi_{2}(t, x)-\omega \phi_{1}(t, x) \leq 0, u_{2}\left[-2 \vartheta \phi_{2}(t, x)-\omega \phi_{1}(t, x)\right]=0 ; \\
& \phi_{1}(t, x) \geq 0, \phi_{2}(t, x) \geq 0 .
\end{aligned}
$$

The conditions above give:

$$
\phi_{1}(t, x)=\frac{\gamma+e^{r t} V_{x}^{1}}{2 \psi} \text { and } \phi_{2}(t, x)=0 .
$$

Note that the authority will decide not to investigate at all.

Substituting $\phi_{1}(t, x)$ and $\phi_{2}(t, x)$ into (3.1) yields

$$
-V_{t}^{1}-\frac{1}{2} \sigma^{2} x^{2} V_{x x}^{1}=e^{-r t}\left[\frac{\gamma\left(\gamma+e^{r t} V_{x}^{1}\right)}{2 \psi}-\varphi x-\frac{\left(\gamma+e^{r t} V_{x}^{1}\right)^{2}}{4 \psi}\right]+V_{x}^{1}\left[\frac{\gamma+e^{r t} V_{x}^{1}}{2 \psi}-\delta x\right] \text {, }
$$


$-V_{t}^{2}-\frac{1}{2} \sigma^{2} x^{2} V_{x x}^{2}=-e^{-r t}\left[\zeta\left(\frac{\gamma+e^{r t} V_{x}^{1}}{2 \psi}\right)^{2}+v x\right]+V_{x}^{2}\left[\frac{\gamma+e^{r t} V_{x}^{1}}{2 \psi}-\delta x\right]$,

$V^{1}(T, x)=-e^{-r T} \eta x$

$V^{2}(T, x)=0$.

\section{Proposition 3.1.}

The system (3.3) admits a solution

$V^{1}(t, x)=e^{-r t}\left[A_{1}(t) x+C_{1}(t)\right]$ and $V^{2}(t, x)=e^{-r t}\left[A_{2}(t) x+C_{2}(t)\right]$,

where

$A_{1}(t), C_{1}(t), A_{2}(t)$ and $C_{2}(t)$ satisfy

$\dot{A}_{1}(t)=(r+\delta) A_{1}(t)+\varphi$,

$\dot{C}_{1}(t)=r C_{1}(t)-\left[\frac{\gamma\left(\gamma+A_{1}(t)\right)}{2 \psi}-\frac{\left[\gamma+A_{1}(t)\right]^{2}}{4 \psi}\right]-A_{1}(t) \frac{\gamma+A_{1}(t)}{2 \psi}$,

$\dot{A}_{2}(t)=(r+\delta) A_{2}(t)+v$,

$\dot{C}_{2}(t)=r C_{2}(t)+\zeta\left(\frac{\gamma+A_{1}(t)}{2 \psi}\right)^{2}-A_{2}(t) \frac{\gamma+A_{1}(t)}{2 \psi}$,

$A_{1}(T)=-\eta, C_{1}(T)=0, A_{2}(T)=0, C_{2}(T)=0$.

Proof. Substituting the relevant derivatives of $V^{1}(t, x)$ and $V^{2}(t, x)$ into (3.3) yields

$$
\begin{aligned}
& r e^{-r t}\left[A_{1}(t) x+C_{1}(t)\right]-e^{-r t}\left[\dot{A}_{1}(t) x+\dot{C}_{1}(t)\right] \\
& =e^{-r t}\left[\frac{\gamma\left(\gamma+A_{1}(t)\right)}{2 \psi}-\varphi x-\frac{\left(\gamma+A_{1}(t)\right)^{2}}{4 \psi}\right]+e^{-r t} A_{1}(t)\left[\frac{\gamma+A_{1}(t)}{2 \psi}-\delta x\right], \\
& r e^{-r t}\left[A_{2}(t) x+C_{2}(t)\right]-e^{-r t}\left[\dot{A}_{2}(t) x+\dot{C}_{2}(t)\right] \\
& =-e^{-r t}\left[\zeta\left(\frac{\gamma+A_{1}(t)}{2 \psi}\right)^{2}+v x\right]+e^{-r t} A_{2}(t)\left[\frac{\gamma+A_{1}(t)}{2 \psi}-\delta x\right] . \\
& r e^{-r T}\left[A_{1}(T) x+C_{1}(T)\right]=-e^{-r T} \eta x,
\end{aligned}
$$


$r e^{-r T}\left[A_{2}(T) x+C_{2}(T)\right]=0$.

For (3.6) to be satisfied, it is required that (3.5) to hold. Hence Proposition 3.1 follows.

System (3.5) forms a block recursive system of differential equations. $A_{1}(t)$ and $A_{2}(t)$ can be solved independent of $C_{1}(t)$ and $C_{2}(t)$. In particular:

$$
\begin{aligned}
& A_{1}(t)=\left(\frac{\varphi}{r+\delta}-\eta\right) e^{(r+\delta)(t-T)}-\frac{\varphi}{r+\delta} \\
& A_{2}(t)=\left(\frac{v}{r+\delta}\right) e^{(r+\delta)(t-T)}-1
\end{aligned}
$$

Note that $A_{2}(t)$ is negative for all $t \in[0, T]$ and $A_{1}(t)$ is negative for all $t \in[0, T]$ if $\eta \leq 0$. This means that an additional unit of criminal record (or experience) $x$ is negatively evaluated by both players at any instant of time. Having a negative shadow price, $x$ is a "bad stock". This is clear since $x$ enters negatively in both objectives.

The game equilibrium strategies of the offender and law enforcer are respectively

$\phi_{1}(t, x)=\frac{1}{2 \psi}\left[\gamma+\left(\frac{\varphi}{r+\delta}-\eta\right) e^{(r+\delta)(t-T)}-\frac{\varphi}{r+\delta}\right]$ and $\phi_{2}(t, x)=0$.

Substituting the game equilibrium strategies into the state dynamics yields:

$$
d x(t)=\left\{\frac{1}{2 \psi}\left[\gamma+\left(\frac{\varphi}{r+\delta}-\eta\right) e^{(r+\delta)(t-T)}-\frac{\varphi}{r+\delta}\right]-\delta x\right\} d t+\sigma x d z(t) .
$$

Equation (3.9) is a linear stochastic differential equation which solution can be expressed as:

$$
\begin{aligned}
x(t)=x_{0} & +\int_{0}^{t}\left\{\frac{1}{2 \psi}\left[\gamma+\left(\frac{\varphi}{r+\delta}-\eta\right) e^{(r+\delta)(t-T)}-\frac{\varphi}{r+\delta}\right]-\delta x(s)\right\} d s \\
& +\int_{0}^{t} \sigma x(s) d z(s) .
\end{aligned}
$$

The stochastic path (3.10) manages to exhibit random elements in its evolution. 


\section{Infinite Horizon and Stationary State}

Now we consider the case when the terminal $T$ approaches infinity. An infinite horizon version of the game (2.1), (2.5) and (2.7) can be specified as:

$$
\begin{aligned}
& \max _{u_{1}} E\left\{\int_{0}^{\infty} e^{-r t}\left[\gamma u_{1}-u_{1} u_{2}-\varphi x-\psi u_{1}^{2}\right] d t\right\} \text { and } \\
& \max _{u_{2}} E\left\{-\int_{0}^{\infty} e^{-r t}\left[\zeta u_{1}^{2}+\vartheta u_{2}^{2}+\nu x+\omega u_{1} u_{2}\right] d t\right\}
\end{aligned}
$$

subject to

$$
d x(t)=\left(u_{1}-\delta x\right) d t+\sigma x d z(t) .
$$

A Nash equilibrium solution for this infinite horizon stochastic differential game can be characterized as:

Theorem 4.1. A pair of feedback strategies $\left\{\phi_{1}^{*}(x) ; \phi_{1}^{*}(x)\right\}$ provides a Nash equilibrium solution to the game (4.1)-(4.2) if there exist suitably smooth functions $V^{i}(x): R \rightarrow R, i \in\{1,2\}$, satisfying the partial differential equations

$$
\begin{aligned}
& r V^{1}(x)-\frac{1}{2} \sigma^{2} x^{2} V_{x x}^{1}(x)=\max _{u_{1}}\left\{\left[u_{1}-u_{1} \phi_{2}(x)-\varphi x-\psi u_{1}^{2}\right]\right. \\
& \left.+V_{x}^{1}(x)\left(u_{1}-\delta x\right)\right\}, \\
& r V_{t}^{2}(x)-\frac{1}{2} \sigma^{2} x^{2} V_{x x}^{2}(x)=\max _{u_{2}}\left\{-\left[\zeta\left(\phi_{1}(x)\right)^{2}+\vartheta u_{2}^{2}+v x+\omega \phi_{1}(x) u_{2}\right]\right. \\
& \left.+V_{x}^{2}(x)\left[\phi_{1}(x)-\delta x\right]\right\} .
\end{aligned}
$$

Proof. Follow the proof of Theorem 2.7.1 in Yeung and Petrosyan (2006).

Performing the indicated maximization yields the conditions:

$$
\begin{aligned}
& \gamma-\phi_{2}(x)-2 \psi \phi_{1}(x)+V_{x}^{1} \leq 0, u_{1}\left[\gamma-\phi_{2}(x)-2 \psi \phi_{1}(x)+V_{x}^{1}\right]=0 ; \\
& -2 \vartheta \phi_{2}(x)-\omega \phi_{1}(x) \leq 0, u_{2}\left[-2 \vartheta \phi_{2}(x)-\omega \phi_{1}(x)\right]=0 ; \\
& \phi_{1}(x) \geq 0, \phi_{2}(x) \geq 0 .
\end{aligned}
$$

The conditions in (4.4) yields: 


$$
\phi_{1}(x)=\frac{\gamma+V_{x}^{1}}{2 \psi} \text { and } \phi_{2}(x)=0 .
$$

Substituting $\phi_{1}(x)$ and $\phi_{2}(x)$ into (4.3) yields

$$
\begin{aligned}
& r V^{1}-\frac{1}{2} \sigma^{2} x^{2} V_{x x}^{1}=\left[\frac{\gamma\left(\gamma+V_{x}^{1}\right)}{2 \psi}-\varphi x-\frac{\left(\gamma+V_{x}^{1}\right)^{2}}{4 \psi}\right]+V_{x}^{1}\left[\frac{\gamma+V_{x}^{1}}{2 \psi}-\delta x\right] \\
& r V_{t}^{2}-\frac{1}{2} \sigma^{2} x^{2} V_{x x}^{2}=-\left[\zeta\left(\frac{\gamma+V_{x}^{1}}{2 \psi}\right)^{2}+v x\right]+V_{x}^{2}\left[\frac{\gamma+V_{x}^{1}}{2 \psi}-\delta x\right]
\end{aligned}
$$

\section{Proposition 4.1.}

The system (4.6) admits a solution

$$
V^{1}(x)=\left[A_{1} x+C_{1}\right] \text { and } V^{2}(x)=\left[A_{2} x+C_{2}\right],
$$

where

$$
\begin{aligned}
& A_{1}=\frac{-\varphi}{r+\delta}, \\
& C_{1}=\left[\frac{\gamma\left(\gamma+A_{1}\right)}{2 \psi r}-\frac{\left(\gamma+A_{1}\right)^{2}}{4 \psi r}\right]+A_{1} \frac{\gamma+A_{1}}{2 \psi r}, \\
& A_{2}=\frac{-v}{r+\delta}, \\
& C_{2}=A_{2} \frac{\gamma+A_{1}}{2 \psi r}-\frac{\zeta}{r}\left(\frac{\gamma+A_{1}}{2 \psi}\right)^{2} .
\end{aligned}
$$

Proof. Follow the proof of Proposition 3.1.

A Nash equilibrium is given by

$$
\phi_{1}(x)=\frac{1}{2 \psi}\left[\gamma-\frac{\varphi}{r+\delta}\right] \text { and } \phi_{2}(x)=0 .
$$

Substituting the game equilibrium strategies into the state dynamics (4.2) yields:

$$
d x(t)=\left[\frac{1}{2 \psi}\left(\gamma-\frac{\varphi}{r+\delta}\right)-\delta x\right] d t+\sigma x(t) d z(t) .
$$

Equation (4.10) is a linear stochastic differential equation with constant coefficients which solution can be expressed as: 


$$
x(t)=x_{0}+\int_{0}^{t}\left[\frac{1}{2 \psi}\left(\gamma-\frac{\varphi}{r+\delta}\right)-\delta x(s)\right] d s+\int_{0}^{t} \sigma x(s) d z(s) .
$$

The stochastic path (4.11) manages to exhibit random elements in its evolution.

The stochastic system (4.10) will generate a stochastic process governed by a joint transition probability density function. This transition probability density function characterizes the possible realizations of $x(t)$.

Let $\mu\left[t, x \mid 0, x_{0}\right]$ denote the transition density function of the vector of $x$ at time $t$, given the initial values $x_{0}$ at time 0 . The function $\mu$ must satisfy the FokkerPlanck 'forward' equation (see Soong 1973 and Yeung (2009)):

$$
\frac{\partial \mu}{\partial t}=-\frac{\partial}{\partial x}\left[\frac{1}{2 \psi}\left(\gamma-\frac{\varphi}{r+\delta}\right)-\delta x\right] \mu+\frac{1}{2} \frac{\partial^{2}}{\partial x^{2}} \sigma^{2} x^{2} \mu \text {. }
$$

A stationary state of the system (4.10) will be characterized by a process which has invariant probability density over time. It implies that the process $x(t)$ approaches a steady state as the transition time $t$ approaches infinity. Specifically

$$
\operatorname{Lim}_{t \rightarrow \infty} \frac{\partial \mu}{\partial t}=0
$$

Therefore, the stationary distribution of $x$ will be represented by a time invariant density function $v(x)$, which in turn will be independent of time $t$ and the initial values of $x_{0}$. In particular, the stationary Fokker-Planck equation can be expressed as:

$$
0=-\frac{\partial}{\partial x}\left[\frac{1}{2 \psi}\left(\gamma-\frac{\varphi}{r+\delta}\right)-\delta x\right] v+\frac{1}{2} \sigma^{2} x^{2} \frac{\partial^{2}}{\partial x^{2}} v .
$$

This differential equation will immediately lead to the result (see Liu (1969) and Soong (1973)):

$$
v(x)=m \exp \left[\int^{x}\left\{\frac{1}{4 \sigma^{2} \tau^{2} \psi}\left(\gamma-\frac{\varphi}{r+\delta}\right)-\frac{\delta}{2 \sigma^{2} \tau}\right\} d \tau\right],
$$


where $m$ is the normalization factor such that $\int_{-\infty}^{\infty} v(x) d x=1$.

The stationary distribution function $v(x)$ characterizes the probability that the state equal $x$ in a steady state.

\section{Concluding Remarks}

This note provides a stochastic version of the Fend-Feichtinger-Tragler dynamic game of offending and law enforcement. Stochastic paths of crimes are derived. This allows the analysis to reflect actual crime statistics which displays randomness in its distribution.

\section{Appendix:}

United States Crime Index Rates Per 100,000 Inhabitants

Forcible Aggravated Larceny- Vehicle

\begin{tabular}{llllllllllllll} 
Year & Population & Total & \multicolumn{2}{c}{ Violent } & Property & Murder Rape & \multicolumn{2}{c}{ Robbery assault } & Burglary & Theft & Theft \\
1960 & $179,323,175$ & $1,887.2$ & 160.9 & $1,726.3$ & 5.1 & 9.6 & 60.1 & 86.1 & 508.6 & $1,034.7$ & 183.0 \\
1961 & $182,992,000$ & $1,906.1$ & 158.1 & $1,747.9$ & 4.8 & 9.4 & 58.3 & 85.7 & 518.9 & $1,045.4$ & 183.6 \\
1962 & $185,771,000$ & $2,019.8$ & 162.3 & $1,857.5$ & 4.6 & 9.4 & 59.7 & 88.6 & 535.2 & $1,124.8$ & 197.4 \\
1963 & $188,483,000$ & $2,180.3$ & 168.2 & $2,012.1$ & 4.6 & 9.4 & 61.8 & 92.4 & 576.4 & $1,219.1$ & 216.6 \\
1964 & $191,141,000$ & $2,388.1$ & 190.6 & $2,197.5$ & 4.9 & 11.2 & 68.2 & 106.2 & 634.7 & $1,315.5$ & 247.4 \\
1965 & $193,526,000$ & $2,449.0$ & 200.2 & $2,248.8$ & 5.1 & 12.1 & 71.7 & 111.3 & 662.7 & $1,329.3$ & 256.8 \\
1966 & $195,576,000$ & $2,670.8$ & 220.0 & $2,450.9$ & 5.6 & 13.2 & 80.8 & 120.3 & 721.0 & $1,442.9$ & 286.9 \\
1967 & $197,457,000$ & $2,989.7$ & 253.2 & $2,736.5$ & 6.2 & 14.0 & 102.8 & 130.2 & 826.6 & $1,575.8$ & 334.1 \\
1968 & $199,399,000$ & $3,370.2$ & 298.4 & $3,071.8$ & 6.9 & 15.9 & 131.8 & 143.8 & 932.3 & $1,746.6$ & 393.0 \\
1969 & $201,385,000$ & $3,680.0$ & 328.7 & $3,351.3$ & 7.3 & 18.5 & 148.4 & 154.5 & 984.1 & $1,930.9$ & 436.2 \\
1970 & $203,235,298$ & $3,984.5$ & 363.5 & $3,621.0$ & 7.9 & 18.7 & 172.1 & 164.8 & $1,084.9$ & $2,079.3$ & 456.8 \\
1971 & $206,212,000$ & $4,164.7$ & 396.0 & $3,768.8$ & 8.6 & 20.5 & 188.0 & 178.8 & $1,163.5$ & $2,145.5$ & 459.8 \\
1972 & $208,230,000$ & $3,961.4$ & 401.0 & $3,560.4$ & 9.0 & 22.5 & 180.7 & 188.8 & $1,140.8$ & $1,993.6$ & 426.1
\end{tabular}




\begin{tabular}{|c|c|c|c|c|c|c|c|c|c|c|c|}
\hline 1973 & $209,851,000$ & $4,154.4$ & 417.4 & $3,737.0$ & 9.4 & 24.5 & 183.1 & 200.5 & $1,222.5$ & $2,071.9$ & 442.6 \\
\hline 1974 & $211,392,000$ & $4,850.4$ & 461.1 & $4,389.3$ & 9.8 & 26.2 & 209.3 & 215.8 & $1,437.7$ & $2,489.5$ & 462.2 \\
\hline 1975 & $213,124,000$ & $5,298.5$ & 487.8 & $4,810.7$ & 9.6 & 26.3 & 220.8 & 231.1 & $1,532.1$ & $2,804.8$ & 473.7 \\
\hline 1976 & $214,659,000$ & $5,287.3$ & 467.8 & $4,819.5$ & 8.7 & 26.6 & 199.3 & 233.2 & $1,448.2$ & $2,921.3$ & 450.0 \\
\hline 1977 & $216,332,000$ & $5,077.6$ & 475.9 & $4,601.7$ & 8.8 & 29.4 & 190.7 & 247.0 & $1,419.8$ & $2,729.9$ & 451.9 \\
\hline 1978 & $218,059,000$ & $5,140.4$ & 497.8 & $4,642.5$ & 9.0 & 31.0 & 195.8 & 262.1 & $1,434.6$ & $2,747.4$ & 460.5 \\
\hline 1979 & $220,099,000$ & $5,565.5$ & 548.9 & $5,016.6$ & 9.8 & 34.7 & 218.4 & 286.0 & $1,511.9$ & $2,999.1$ & 505.6 \\
\hline 1980 & $225,349,264$ & $5,950.0$ & 596.6 & $5,353.3$ & 10.2 & 36.8 & 251.1 & 298.5 & $1,684.1$ & $3,167.0$ & 502.2 \\
\hline 1981 & $229,146,000$ & $5,858.2$ & 594.3 & $5,263.8$ & 9.8 & 36.0 & 258.7 & 289.7 & $1,649.5$ & $3,139.7$ & 474.7 \\
\hline 1982 & $231,534,000$ & $5,603.7$ & 571.1 & $5,032.5$ & 9.1 & 34.0 & 238.9 & 289.1 & $1,488.8$ & $3,084.9$ & 458.9 \\
\hline 1983 & $233,981,000$ & $5,175.0$ & 537.7 & $4,637.3$ & 8.3 & 33.7 & 216.5 & 279.2 & $1,337.7$ & $2,869.0$ & 430.8 \\
\hline 1984 & $236,158,000$ & $5,031.3$ & 539.2 & $4,492.1$ & 7.9 & 35.7 & 205.4 & 290.2 & $1,263.7$ & $2,791.3$ & 437.1 \\
\hline 1985 & $238,740,000$ & $5,207.1$ & 556.6 & $4,650.5$ & 8.0 & 37.1 & 208.5 & 302.9 & $1,287.3$ & $2,901.2$ & 462.0 \\
\hline 1986 & $240,132,887$ & $5,480.4$ & 620.1 & $4,881.8$ & 8.6 & 38.1 & 226.0 & 347.4 & $1,349.8$ & $3,022.1$ & 509.8 \\
\hline 1987 & $243,400,000$ & $5,550.0$ & 609.7 & $4,940.3$ & 8.3 & 37.4 & 212.7 & 351.3 & $1,329.6$ & $3,081.3$ & 529.5 \\
\hline 1988 & $245,807,000$ & $5,664.2$ & 637.2 & $5,027.1$ & 8.4 & 37.6 & 220.9 & 370.2 & $1,309.2$ & $3,134.9$ & 582.9 \\
\hline 1989 & $248,239,000$ & $5,741.0$ & 663.1 & $5,077.9$ & 8.7 & 38.1 & 233.0 & 383.4 & $1,276.3$ & $3,171.3$ & 630.4 \\
\hline 1990 & $248,709,873$ & $5,820.3$ & 731.8 & $5,088.5$ & 9.4 & 41.2 & 257.0 & 424.1 & $1,235.9$ & $3,194.8$ & 657.8 \\
\hline 1991 & $252,177,000$ & $5,897.8$ & 758.1 & $5,139.7$ & 9.8 & 42.3 & 272.7 & 433.3 & $1,252.0$ & $3,228.8$ & 658.9 \\
\hline 1992 & $255,082,000$ & $5,660.2$ & 757.5 & $4,902.7$ & 9.3 & 42.8 & 263.6 & 441.8 & $1,168.2$ & $3,103.0$ & 631.5 \\
\hline 1993 & $257,908,000$ & $5,484.4$ & 746.8 & $4,737.7$ & 9.5 & 41.1 & 255.9 & 440.3 & $1,099.2$ & $3,032.4$ & 606.1 \\
\hline 1994 & $260,341,000$ & $5,373.5$ & 713.6 & $4,660.0$ & 9.0 & 39.3 & 237.7 & 427.6 & $1,042.0$ & $3,026.7$ & 591.3 \\
\hline 1995 & $262,755,000$ & $5,274.9$ & 684.5 & $4,591.3$ & 8.2 & 37.1 & 220.9 & 418.3 & 987.1 & $3,043.8$ & 560.4 \\
\hline 1996 & $265,284,000$ & $5,087.6$ & 636.6 & $4,451.0$ & 7.4 & 36.3 & 201.9 & 390.9 & 945.0 & $2,980.3$ & 525.7 \\
\hline 1997 & $267,637,000$ & $4,927.3$ & 611.0 & $4,316.3$ & 6.8 & 35.9 & 186.1 & 382.1 & 919.6 & $2,891.8$ & 505.7 \\
\hline 1998 & $270,296,000$ & $4,615.5$ & 566.4 & $4,049.1$ & 6.3 & 34.4 & 165.2 & 360.5 & 862.0 & $2,728.1$ & 459.0 \\
\hline 1999 & $272,690,813$ & $4,266.5$ & 523.0 & $3,743.6$ & 5.7 & 32.8 & 150.1 & 334.3 & 770.4 & $2,550.7$ & 422.5 \\
\hline
\end{tabular}




$\begin{array}{llllllllllll}2000 & 281,421,906 & 4,124.8 & 506.5 & 3,618.3 & 5.5 & 32.0 & 145.0 & 324.0 & 728.8 & 2,477.3 & 412.2 \\ 2001 & 285,317,559 & 4,162.6 & 504.5 & 3,658.1 & 5.6 & 31.8 & 148.5 & 318.6 & 741.8 & 2,485.7 & 430.5 \\ 2002 & 287,973,924 & 4,125.0 & 494.4 & 3,630.6 & 5.6 & 33.1 & 146.1 & 309.5 & 747.0 & 2,450.7 & 432.9 \\ 2003 & 290,690,788 & 4,067.0 & 475.8 & 3,591.2 & 5.7 & 32.3 & 142.5 & 295.4 & 741.0 & 2,416.5 & 433.7 \\ 2004 & 293,656,842 & 3,977.3 & 463.2 & 3,514.1 & 5.5 & 32.4 & 136.7 & 288.6 & 730.3 & 2,362.3 & 421.5 \\ 2005 & 296,507,061 & 3,900.5 & 469.0 & 3,431.5 & 5.6 & 31.8 & 140.8 & 290.8 & 726.9 & 2,287.8 & 416.8 \\ 2006 & 299,398,484 & 3,808.1 & 473.6 & 3,334.5 & 5.7 & 30.9 & 149.4 & 287.5 & 729.4 & 2,206.8 & 398.4 \\ 2007 & 301,621,157 & 3,730.4 & 466.9 & 3,263.5 & 5.6 & 30.0 & 147.6 & 283.8 & 722.5 & 2,177.8 & 363.3 \\ 2008 & 304,059,724 & 3,667.0 & 454.5 & 3,212.5 & 5.4 & 29.3 & 145.3 & 274.6 & 730.8 & 2,167.0 & 314.7\end{array}$

Source: The Disaster Center

References

Fent, T., Feichtinger, G. and Tragler, G. (2002) A Dynamic Game of Offending and Law Enforcement. International Game Theory Review, 4(1), 71-89.

Greenwood, P.W., Rydell, C.P., Abrahamse, A.F., Chiesa, J., Model, K. and Klein, S. (1994) Three Strikes and You are Out: Estimated Benefits and Costs of California's New Mandatory Sentencing Law. MR-509-RC, The RAND Corporation.

Liu, S.C. (1969) Solution of Fokker-Planck equation with applications in non-linear random vibration. Bell System Technical Journal, 48, 2031-2051.

Soong, T.T. (1973) Random Differential Equations in Science and Engineering, Academic Press, New York.

Yeung, D.W.K. (2009) An Explicit Density Function for a Generalized Stochastic Foodchain of the Lotka-Volterra-Yeung Type, Stochastic Analysis and Applications, 27(1), 16-23.

Yeung, D.W.K. and Petrosyan, L.A. (2006): Cooperative Stochastic Differential Games, New York: Springer-Verlag. 